\title{
Lichenoid Keratosis-Like Melanoma: A Clinically and Histopathologically Challenging Lesion
}

\author{
Maral Sahil ${ }^{a}$ Yasmine Lucile Ibrahim ${ }^{b}$ Gürkan Kayaa, b \\ aDepartment of Dermatology, University Hospital of Geneva, Geneva, Switzerland; \\ ${ }^{b}$ Department of Clinical Pathology, University Hospital of Geneva, Geneva, Switzerland
}

\section{Keywords}

Regressive melanoma · Lichenoid keratosis · Gray dots

\begin{abstract}
Regressive melanoma with lichenoid tissue reaction may be clinically and histopathologically challenging. A 73-year-old male visited our clinic for a general skin checkup. We noticed a red papule on the right side of his back. On dermatoscopy, scattered grayish dots were observed. The differential diagnosis included lichenoid keratosis and melanoma. Histopathological examination of the excised lesion showed a regressive melanoma with a histopathological pattern of lichenoid keratosis.

(C) 2020 The Author(s)

Published by S. Karger AG, Basel
\end{abstract}

\section{Background}

A 73-year-old male with a history of hypertensive nephrosclerosis leading to terminal chronic renal insufficiency and renal allograft underwent his annual skin checkup in our clinic. A red papule was noticed on the right side of the back (Fig. 1). On dermatoscopy, we observed grayish dots scattered in the center and the periphery of the lesion. The clinical differential diagnosis was lichenoid keratosis or malignant melanoma. The lesion was surgically removed.

\section{Method}

The biopsy material obtained from the patient was fixed in formalin, embedded in paraffin, cut at $5 \mu \mathrm{m}$, and stained with hematoxylin-eosin. 

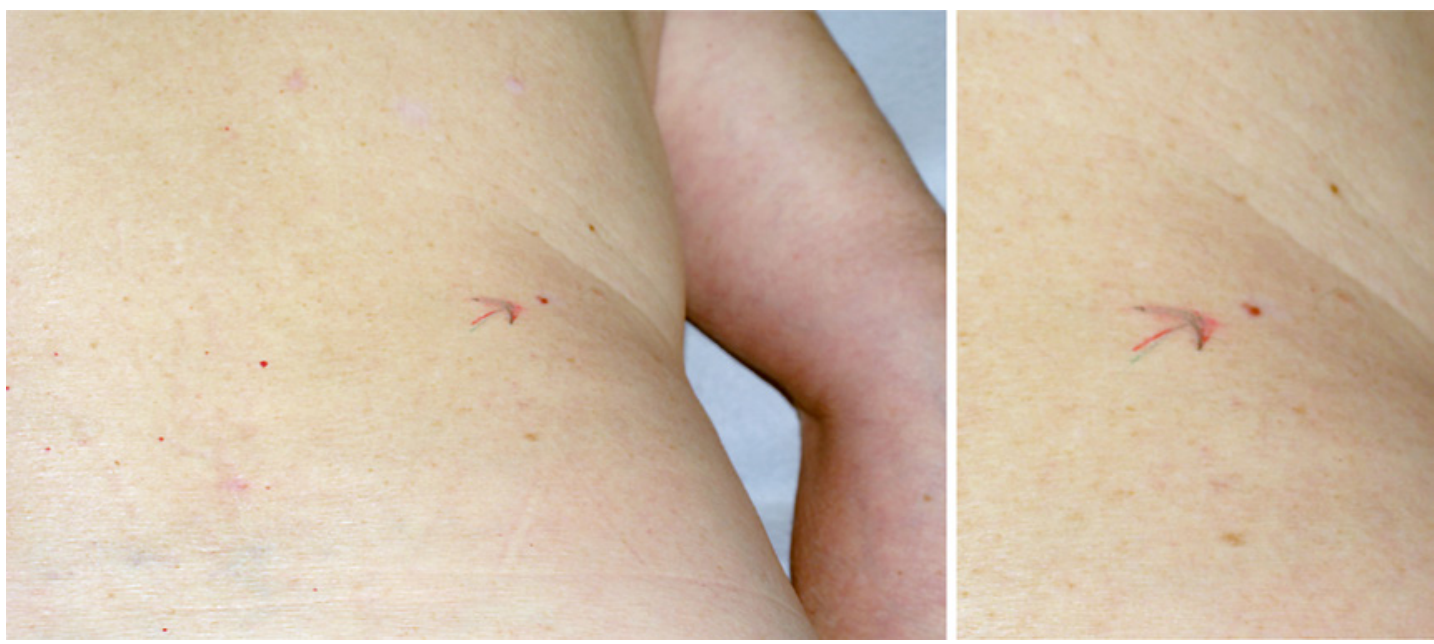

Fig. 1. Red papule on the right side of the back.
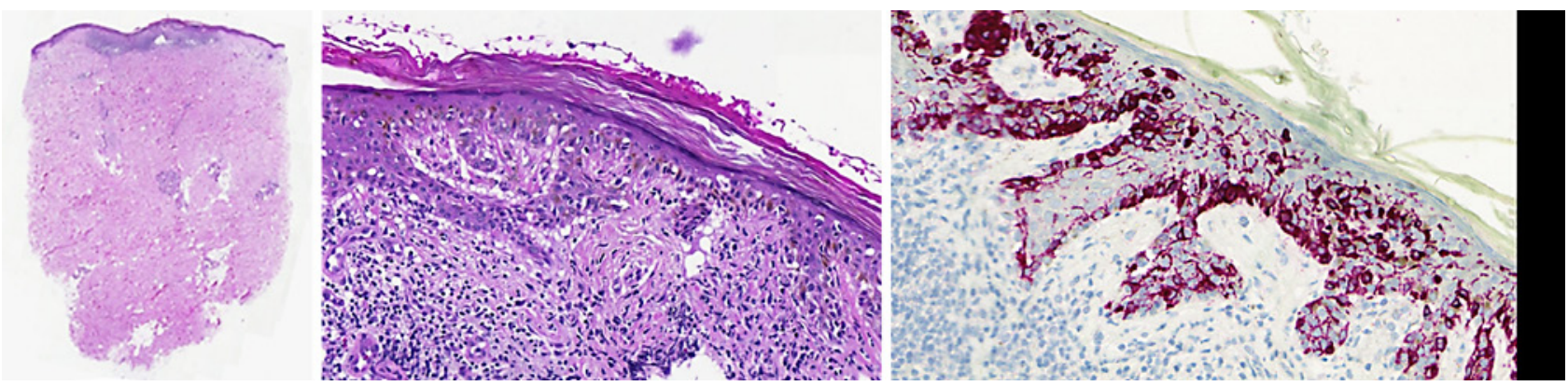

Fig. 2. Dermal lymphocytic infiltrate with a lichenoid pattern and proliferation of atypical melanocytes showing pagetoid spread within the epidermis, confirmed by Melan-A staining.

\section{Results}

Histopathological examination revealed a dense dermal lymphocytic infiltrate with a lichenoid pattern containing melanophages, vacuolar degeneration and necrosis of basal keratinocytes, and a proliferation of atypical melanocytes showing pagetoid spread within the epidermis, confirmed by Melan-A staining (Fig. 2). A diagnosis of regressive melanoma with a histopathological pattern of lichenoid keratosis was made.

\section{Discussion}

Lichenoid keratosis or lichen planus-like keratosis has been proposed to be a regressive epidermal lesion such as solar lentigo or seborrheic keratosis. Usually, this lesion is localized on the trunk, but less frequently on the extremities, head, and neck. The clinical aspect is a red-brown papule. The most common dermatoscopic finding is a granular pattern (reddishbrown, bluish-gray, or whitish-gray granules) [1].

Regressive melanoma can be clinically and dermatoscopically indistinguishable from lichenoid keratosis. The presence of a single lesion showing clinical features of lichenoid keratosis should raise the concern of a melanoma undergoing regression [2, 3]. 
Multiple blue-grey dots are found in $45 \%$ of invasive melanoma and have a specificity of $91 \%$. It corresponds histopathologically to melanophages in the early-intermediate phase of regression. The other differential diagnoses are basal cell carcinoma, seborrheic keratosis, actinic keratosis, Bowen's disease, and invasive squamous cell carcinoma.

Histopathological examination should be thoroughly performed and include immunohistochemistry to search for the potential signs of melanoma, such as pagetoid spread of atypical melanocytes within the epidermis [4].

Immunohistochemical analysis is necessary to avoid missing a melanoma showing clinical and histopathological features of lichenoid keratosis.

\section{Statement of Ethics}

This case report was prepared in compliance with all ethical and confidentiality guidelines and principles. The authors obtained informed oral consent from the patient's wife as it was not practical to have it in writing because of her advanced age. The authors consulted the President of the Ethical Commission on Human Research in Geneva who approved the consent procedure.

\section{Disclosure Statement}

The authors have no conflicts of interest to declare.

\section{Funding Sources}

No funding was received.

\section{Author Contributions}

M.S. and Y.L.I. contributed to the acquisition, analysis, and interpretation of the data for the work, participated in drafting the work, approved the final version to be published, and agreed to be accountable for all aspects of the work in ensuring that questions related to the accuracy or integrity of any part of the work are appropriately investigated and resolved. G.K. made substantial contributions to the conception and design of the work and to the acquisition, analysis, and interpretation of the data for the work; participated in drafting the work, revising it critically for important intellectual content, approved the final version to be published, and agreed to be accountable for all aspects of the work in ensuring that questions related to the accuracy or integrity of any part of the work are appropriately investigated and resolved.

\section{References}

1 Zaballos P, Blazquez S, Puig S, Salsench E, Rodero J, Vives JM, et al. Dermoscopic pattern of intermediate stage in seborrhoeic keratosis regressing to lichenoid keratosis: report of 24 cases. Br J Dermatol. 2007 Aug; 157(2): 266-72.

2 Raptoulis G, Spencer R, Einstein B, Oliviero M, Braun R, Rabinovitz H. Lichen planus-like keratosis of the face: a simulator of melanoma in situ. Dermatol Surg. 2007 Jul;33(7):854-6.

3 Moscarella E, Zalaudek I, Pellacani G, Eibenschutz L, Catricalà C, Amantea A, et al. Lichenoid keratosis-like melanomas. J Am Acad Dermatol. 2011 Sep;65(3):e85-7.

4 Dalton SR, Fillman EP, Altman CE, Gardner TL, Davis TL, Bastian BC, et al. Atypical junctional melanocytic proliferations in benign lichenoid keratosis. Hum Pathol. 2003 Jul;34(7):706-9. 